\title{
Intravesical treatment of chemotherapeutic agents sensitizes bacillus Calmette-Guerin by the modulation of the tumor immune environment
}

\author{
SHUNTA HORI, MAKITO MIYAKE, YOSHIHIRO TATSUMI, YOSUKE MORIZAWA, YASUSHI NAKAI, \\ SAYURI ONISHI, KENTA ONISHI, KOTA IIDA, DAISUKE GOTOH, YOSHITAKA ITAMI, \\ NOBUMICHI TANAKA and KIYOHIDE FUJIMOTO
}

Department of Urology, Nara Medical University, Kashihara, Nara 634-8522, Japan

Received October 6, 2018; Accepted December 17, 2018

DOI: 10.3892/or.2019.6965

\begin{abstract}
Intravesical treatment with bacillus Calmette-Guerin (BCG) is the most common treatment for preventing progression and recurrence of non-muscle invasive bladder cancer. Our previous study using the N-butyl-N-(4-hydroxybutyl) nitrosamine (BBN)-induced orthotopic bladder cancer model demonstrated that intravesical treatment with mitomycin $\mathrm{C}$ (MMC) and adriamycin (ADM) suppressed pro-tumoral immunity, including the aggregation of tumor-associated macrophages (TAMs) and regulatory T cells (Tregs) in the tumor microenvironment. Previous evidence supports the association of resistance to intravesical treatment of BCG with TAMs and Tregs. In the present study, we investigated the antitumoral efficacy of sequential intravesical treatments with chemotherapeutic agents and BCG in a BBN-induced orthotopic bladder cancer model. Thirty-six C57BL/6J mice bearing bladder cancer were randomly divided into six treatment
\end{abstract}

Correspondence to: Dr Kiyohide Fujimoto, Department of Urology, Nara Medical University, 840 Shijo-Cho, Kashihara, Nara 634-8522, Japan

E-mail: kiyokun@naramed-u.ac.jp

Abbreviations: UCB, urothelial carcinoma of the bladder; NMIBC, non-muscle invasive bladder cancer; BCG, bacillus Calmette-Guerin; MMC, mitomycin C; ADM, adriamycin; TAM, tumor-associated macrophage; Treg, regulatory T cell; IL, interleukin; G-CSF, granulocyte-colony stimulating factor; BBN, N-butyl-N-(4-hydroxybutyl) nitrosamine; PBS, phosphate-buffered saline; H\&E, hematoxylin and eosin; IHC, immunohistochemistry; ELISA, enzyme-linked immunosorbent assay; NK, natural killer; HPF, high power field; CTL, cytotoxic T lymphocyte; Th, T-helper; IFN, interferon; TNF, tumor necrosis factor; TRAIL, tumor necrosis factor-related apoptosis-inducing ligand; Fas, fatty-acid synthetase; DFI, disease-free interval; MDSC, myeloid-derived suppressor cell

Key words: bacillus Calmette-Guerin, bladder cancer, intravesical chemotherapy, sequential treatment, immunoreaction, immunomodulation groups as follows: Control, BCG, MMC, ADM, MMC-BCG and ADM-BCG. Intravesical treatment was performed once a week for six weeks. One week after the completion of intravesical treatment, bladder and blood were harvested. MMC-BCG and ADM-BCG were more effective antitumor activities than BCG monotherapy. Bladders were subjected to immunohistochemical analysis and revealed that intravesical BCG treatment combined with MMC/ADM promoted the local recruitment of NK cells to the bladder as effectively as BCG monotherapy and reduced TAMs and Tregs in the bladder. Interleukin (IL)-17 and granulocyte-colony stimulating factor (G-CSF) in serum were analyzed by enzyme-linked immunosorbent assay and these levels were revealed to be elevated in mice treated with sequential treatments similar to levels following monotherapy with MMC and ADM. Our findings indicated that intravesical sequential treatment could suppress the resistance to BCG through the enhancement of antitumor immunity (induction of NK cells) and inhibition of pro-tumoral immunity (reduction of TAMs and Tregs). Systemic changes in IL-17 and G-CSF may be involved in topical immunomodulation. Further studies including clinical trials may be required to establish an appropriate strategy based on the immunomodulation of the tumor microenvironment.

\section{Introduction}

Urothelial carcinoma of the bladder (UCB) is the fourth most common cancer among men and the second most frequent malignancy of the urogenital tract. It is estimated that globally 76,960 patients were newly diagnosed with UCB and 16,390 patients succumbed to the disease in 2016 (11,820 men and 4,570 women) (1). In Japan, the estimated number of new cases and deaths from UCB in 2017 were 21,000 and 8,800 (6,100 men and 2,700 women), respectively (2). Approximately $75 \%$ of UCB cases are diagnosed as non-muscle invasive bladder cancer (NMIBC) consisting of tumors staged as Ta, $\mathrm{T} 1$ and carcinoma in situ (3). Transurethral resection of the bladder tumor, followed by the administration of adjuvant intravesical treatment with bacillus Calmette-Guerin (BCG) or chemotherapeutic agents such as mitomycin C (MMC) and adriamycin (ADM), is the gold-standard treatment for 
NMIBC (4). Despite improved management for decreasing the recurrence rate and prolonging the progression-free interval, NMIBC exhibits significant potential of recurrence intravesically and progression to muscle-invasive bladder cancer $(5,6)$. Although intravesical treatment with BCG represents the most effective and common form of adjuvant therapy for high risk NMIBC, BCG-failure NMIBC is the one of main problems in the management of UCB (7-9). Thus, clinical management of high-risk NMIBC remains challenging and further advancements in treatment initiation and maintenance are urgent.

Intravesical sequential treatment with BCG and chemotherapeutic agents could be a strategy to improve outcomes for patients with BCG-failure NMIBC (10-18). Previous studies have revealed that intravesical sequential treatment with $\mathrm{BCG}$ and chemotherapeutic agents (MMC and ADM) decreased the recurrence rates of NMIBC, compared with BCG treatment alone (15-17). The roles of chemotherapeutic agents in sequential treatment have direct antitumor effects, which leads to a reduction in tumor cells and tissue-scarifying of the bladder surface. BCG binds to the urothelial lining and tumor cells via fibronectin attachment protein on bacilli and then elicits a non-specific immune response within the bladder wall, involving the activation of multiple types of immune cells and cytokines. Intravesical chemotherapies damage the bladder wall, enhancing the expression of fibronectin, and rendering BCG easier to attach, resulting in the enhanced antitumor activity of BCG (12-14,16,19-21).

Our previous study on 154 NMIBCs revealed that high counts of tumor-associated macrophages (TAMs) and regulatory T cells (Tregs) were associated with shorter recurrence-free survival, while high Treg counts were an independent predictor for recurrence. The presence of TAM and Treg in the tumor microenvironment led to a poor response to the treatment with BCG (22). In an animal model, intravesical treatment with MMC and ADM also caused a reduction of TAMs and Tregs in the tumor microenvironment and induced systemic changes to cytokines, including interleukin (IL)-17 and granulocyte-colony stimulating factor (G-CSF) (23). There are limited studies describing details regarding immunoreactions induced by treatments with BCG and chemotherapeutic agents, and their efficacy from an immunological standpoint. The aim of the present study was to immunologically evaluate the efficacy of intravesical chemotherapeutic agents, MMC or ADM, combined with BCG using an N-butyl-N-(4-hydroxybutyl) nitrosamine (BBN)-induced orthotopic bladder cancer model.

\section{Materials and methods}

Animals. Animal care was in compliance with the recommendations of The Guide for Care and Use of Laboratory Animals (National Research Council) and approval for the animal studies was obtained from the Ethics Committee on Animal Research of Nara Medical University (reference no. 11649). Thirty-six 6-week-old female C57BL/6J mice weighing $25 \mathrm{~g}$ were obtained from Oriental Bio Service (Kyoto, Japan). They were kept in a temperature- and humidity-controlled room, with a 12-h light/dark cycle and food were given ad libitum.

Reagents. BBN (B0938; Tokyo Chemical Industry, Tokyo, Japan) was used to prepare an orthotopic murine bladder cancer model. To treat mice bearing BBN-induced bladder cancer with intravesical instillation, the following agents, which are commonly used in a clinical setting for NMIBC, were prepared: BCG (Nippon Kayaku, Tokyo, Japan), MMC (Kyowa Hakko Kirin, Tokyo, Japan) and ADM (Wako, Osaka, Japan). Each agent was diluted in sterile saline solution according to the manufacturer's instructions, and the dosage of each agent was determined by our previous study (23). Sterile phosphate-buffered saline (PBS) was used as a control.

Murine orthotopic bladder cancer model and intravesical treatment. Fig. 1A displays a schematic representation of the study. After allowing mice to acclimate to our facility for a week, they received $0.05 \% \mathrm{BBN}$ in drinking water, continuously for 16 weeks, to induce the development of NMIBC as previously described (23). Mice were then allowed to resume drinking $\mathrm{BBN}$-free water after 17 weeks and were randomly divided into six groups ( $\mathrm{N}=6 \mathrm{mice} / \mathrm{group})$ as follows: Control (PBS), BCG $(10 \mu \mathrm{g} / \mathrm{kg}), \mathrm{MMC}(5 \mu \mathrm{g} / \mathrm{kg})$, ADM $(5 \mu \mathrm{g} / \mathrm{kg})$, MMC-BCG (5 and $10 \mu \mathrm{g} / \mathrm{kg}$, respectively), and ADM-BCG (5 and $10 \mu \mathrm{g} / \mathrm{kg}$, respectively). Intravesical treatment was initiated from week 17 and administered once a week for six weeks. The treatment schedule was determined based on our previous study that suggested that intravesical treatment with MMC and ADM suppressed the expression of TAMs and Tregs (23). Our hypothesis was that the administration of MMC and ADM prior to BCG treatment could modulate the tumor microenvironment to produce conditions allowing BCG to produce a greater effect. Intravesical instillation regimens of each group are presented in Fig. 1A. In regimens containing $\mathrm{BCG}$, mice were treated with $\mathrm{BCG}$ at weeks 2, 3, 5 and 6. In regimens containing MMC or ADM, mice were treated with MMC or ADM at weeks 1 and 4. Fig. 1B illustrates the procedure of catheterization and occlusion with a purse-string suture. All bladder instillations were performed under anesthesia with isoflurane, whereby a 24-gauge Teflon angiocatheter was placed into the bladder via the urethra. Urine was completely drained from the bladder, and then PBS, BCG, MMC and/or ADM were delivered by transurethral instillation through the catheter and allowed to dwell in the bladder by occlusion of the urethra with a purse-string suture. After $2 \mathrm{~h}$, the suture was removed and mice were stimulated to expel bladder contents. One week after the last intravesical treatment, all mice were euthanized by exsanguination under anesthesia with isoflurane and tissues (bladders and whole blood by cardiac puncture) were harvested for the following experiments. Resected bladder weights were assessed by an electronic precision weighing scale and then examined by hematoxylin and eosin (H\&E) staining and immunohistochemical (IHC) staining analysis. Treatment-related changes in IL-17 and G-CSF in sera were evaluated by enzyme-linked immunosorbent assay (ELISA)-based analysis. The reason why we focused on IL-17 and G-CSF is that our previous study revealed systemic changes in these two cytokines (23).

IHC staining analysis. Tumors were examined by IHC staining analysis as previously described $(24,25)$. All resected bladders were filled with $150 \mu 1$ of $10 \%$ neutral buffered formalin, and entire specimens were placed in $10 \%$ neutral 


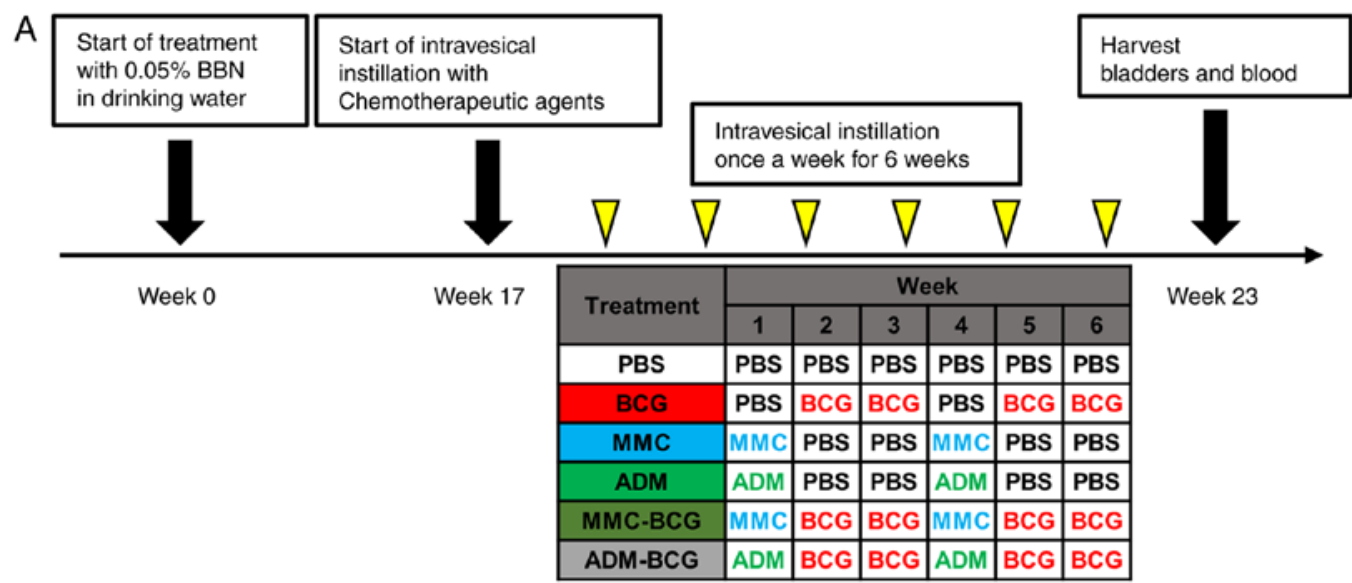

B

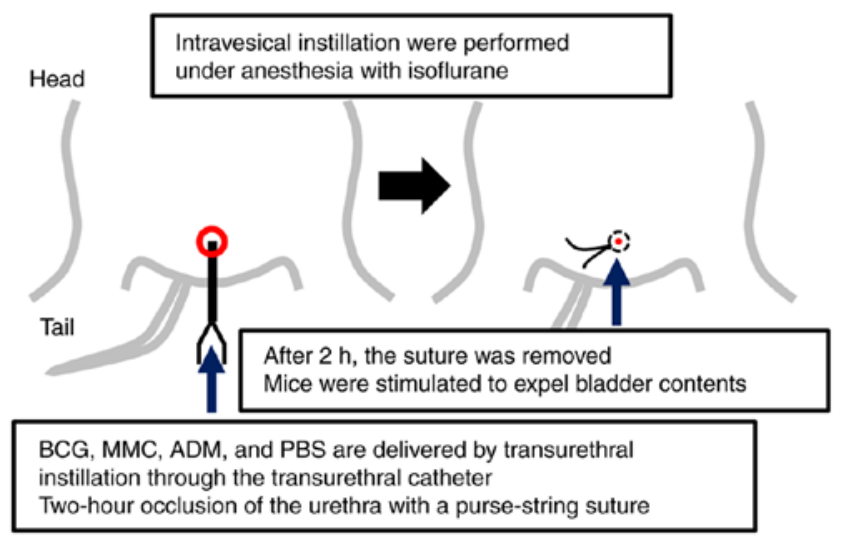

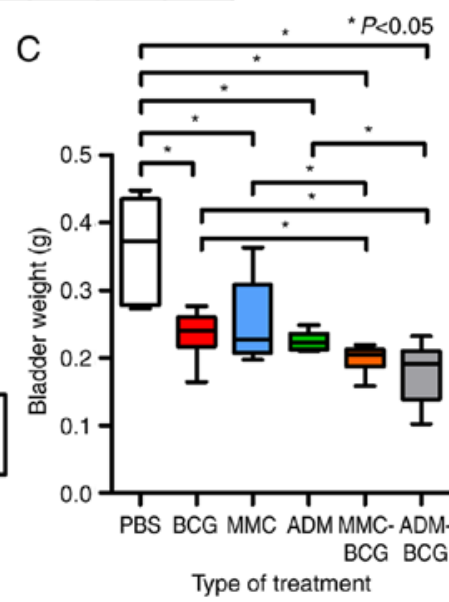

Type of treatment

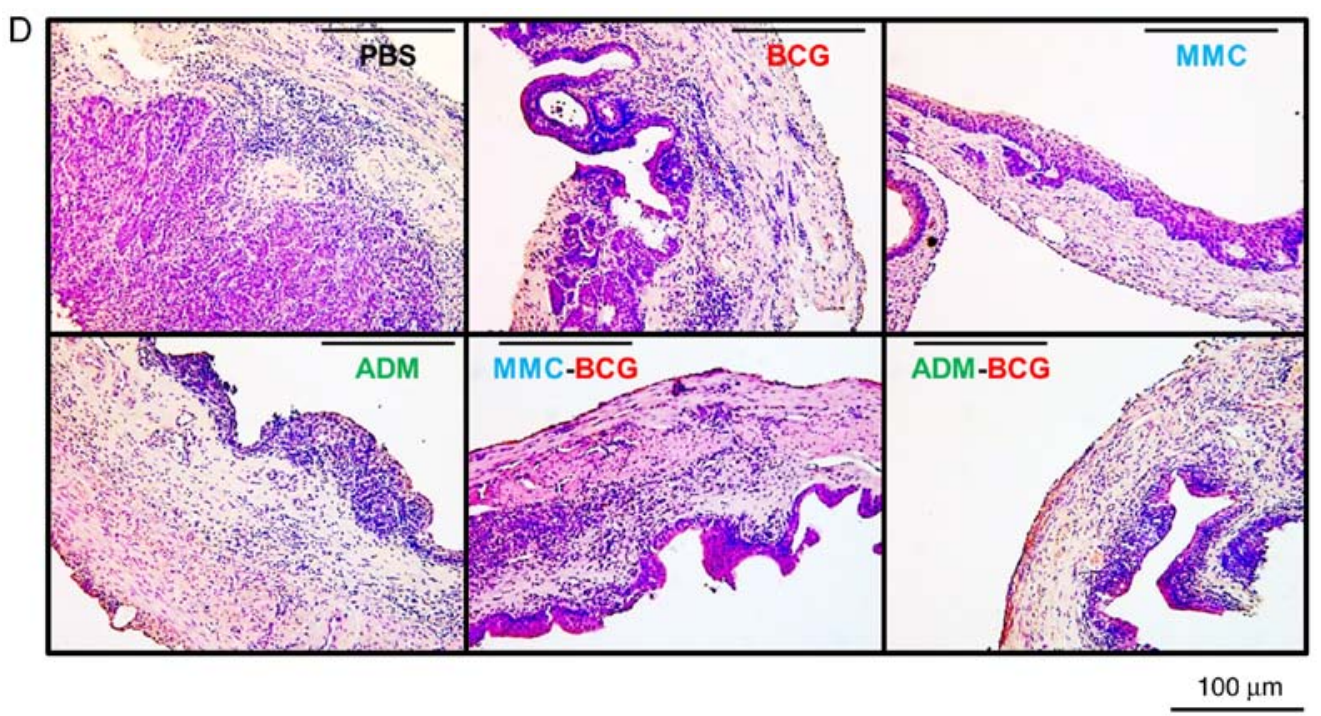

Figure 1. Study treatment schema and resected bladder tissues. (A) Schematic diagram illustrating the study workflow. Mice were administered $0.05 \%$ BBN in drinking water continuously for 16 weeks and were then randomly divided into six groups ( $\mathrm{N}=6 /$ group). Starting at week 17 , mice were treated once a week for 6 weeks with PBS, BCG, MMC, or ADM. One week after the last treatment, mice were euthanized for harvest of the bladder and whole blood by cardiac puncture. Anti-CD56, CD204, and Foxp3 antibodies were used to evaluate natural killer cells, tumor-associated macrophages, and regulatory T cells, respectively, in resected bladder samples. Serum was used to perform enzyme-linked immunosorbent assays. (B) Illustration revealing the procedure of intravesical instillation and occlusion of the urethra with a purse-string suture. All intravesical treatments were performed under anesthesia with isoflurane, and all drugs were delivered by transurethral instillation through a catheter and allowed to dwell in the bladder. After $2 \mathrm{~h}$, sutures were removed and mice were allowed to urinate. (C) Comparison between resected bladder weights of each treatment group. All intravesical treatments had antitumor activity similar to BCG. The intravesical combination treatment with BCG and MMC or ADM caused significant bladder weight loss compared with that with BCG alone or the chemotherapeutic agent alone. "P<0.05. (D) Representative images of hematoxylin and eosin-stained bladder samples from each group. BBN-induced bladder cancer was observed in all resected bladders. BBN, N-butyl-N-(4-hydroxybutyl) nitrosamine; PBS, phosphate-buffered saline; BCG, bacillus Calmette-Guerin; MMC, mitomycin C; ADM, adriamycin .

buffered formalin. Bladders in formalin were embedded in paraffin and then subjected to IHC staining for cell surface and immunological markers, CD56 [natural killer (NK) cell], CD204 (M2 macrophage, known as TAM), and Foxp3 
Table I. Summary of immunohistochemical staining and ELISA assay of serum.

Immunohistochemical staining analysis of the treated bladder (vs. the control) ELISA of serum (vs. the control)

\begin{tabular}{lllllc}
\hline Treatment & CD56 & CD204 & Foxp3 & IL-17 & G-CSF \\
\hline BCG & Up & NS & NS & Up & Up \\
MMC & Down & Down & Down & NS & NS \\
ADM & NS & Down & Down & NS & Up \\
MMC-BCG & Up & Down & NS & Up & NS \\
ADM-BCG & Up & Down & Down & Up & Up \\
\hline
\end{tabular}

ELISA, enzyme-linked immunosorbent assay; BCG, bacillus Calmette-Guerin; MMC, mitomycin C; ADM, adriamycin; IL, interleukin; G-CSF, granulocyte-colony stimulating factor; NS, not significant compared with the control $(\mathrm{P}>0.05)$; Up, significantly increased compared with the control $(\mathrm{P}<0.05)$; Down, significantly decreased compared with the control $(\mathrm{P}<0.05)$.

(Treg). Paraffin blocks were cut and placed on SuperFrost Plus microslides (Thermo Fisher Scientific, Inc., Yokohama, Japan). Sections were deparaffinized and antigen retrieval was carried out in citric acid buffer ( $\mathrm{pH}$ 6.0) using an autoclave. IHC staining was performed using a Histofine ABC kit (Nichirei Biosciences, Tokyo, Japan) according to the manufacturer's instructions. Briefly, slides were incubated overnight at $4{ }^{\circ} \mathrm{C}$ with mouse monoclonal antibodies against CD56 (1:500 dilution; cat. no. MA1-70100; Thermo Fisher Scientific, Inc.), CD204 (1:2,000 dilution; cat. no. KT022; TransGenic, Inc., Kobe, Japan), and Foxp3 (1:500 dilution; ab20034; Abcam, Cambridge, UK). The slides were counterstained with Mayer's hematoxylin, dehydrated, and sealed with a cover slide. In each case, positive cells were evaluated by two investigators (MM and YT), who were blinded to the information pertaining to the treatment. Positive cells from each specimen were counted from a minimum of four randomly selected fields per high power field (HPF; magnification, $\mathrm{x} 400 ; 0.0625 \mu \mathrm{m}^{2}$ ) and compared with the control by calculating the average number of cells.

Assessment of serum IL-17 and G-CSF by ELISA. Serum was collected in tubes, centrifuged at $10,000 \mathrm{x} \mathrm{g}$ for $15 \mathrm{~min}$, and the supernatant was stored at $-80^{\circ} \mathrm{C}$. All 36 serum samples were thawed just before use and analyzed for their concentrations of IL-17 (BMS6001; Affymetrix eBioscience; Thermo Fisher Scientific, Inc.) and G-CSF (ELM-GCSF; RayBiotech, Norcross, GA, USA). Samples were developed with horseradish peroxidase-conjugated secondary antibodies. After adding the substrate and stop solution, a Tecan microplate reader (Tecan, San Jose, CA, USA) was used to assess the absorbance at $450 \mathrm{~nm}$.

Statistical analysis. Statistical analyses and figure plotting were performed using GraphPad Prism 5.0 (GraphPad Software, Inc., San Diego, CA, USA). Data are expressed as bar charts or box plots and the Student's t-test or the Mann-Whitney U test was applied for statistical analysis, as appropriate. A P-value $<0.05$ was considered to indicate a statistically significant result in all analyses.

\section{Results}

Antitumor effects of intravesical treatment with BCG, MMC, $A D M$ and the sequential treatment. All mice were intravesically treated for six weeks with PBS, BCG, MMC, ADM, MMC-BCG,or ADM-BCG according to the regimens displayed in Fig. 1A. All treatments were well tolerated, with no body weight loss except minor hematuria. A week after the termination of treatment, significant bladder weight loss was observed in the BCG, MMC, ADM, MMC-BCG, and ADM-BCG treatment groups compared with the control $(\mathrm{P}=0.0043, \mathrm{P}=0.041$, $\mathrm{P}=0.0023, \mathrm{P}=0.0021, \mathrm{P}=0.0020$, respectively; Fig. 1C). Moreover, significant differences were observed between the bladder weights of mice receiving BCG monotherapy or MMC/ADM monotherapy and sequential treatments with BCG and MMC/ADM (BCG vs. MMC-BCG, $\mathrm{P}=0.042$; BCG vs. $\mathrm{ADM}-\mathrm{BCG}, \mathrm{P}=0.026$; $\mathrm{MMC}$ vs. $\mathrm{MMC}-\mathrm{BCG}, \mathrm{P}=0.041$; $\mathrm{ADM}$ vs. $\mathrm{ADM}-\mathrm{BCG}, \mathrm{P}=0.0040$ ). These results indicated that the antitumor effects were increased by combining BCG with chemotherapeutic agents. Fig. 1D displays representative H\&E staining images for each treatment group and the development of NMIBC was confirmed in all samples.

Features of immune-related cells induced by intravesical sequential treatment with BCG and chemotherapeutic agents. To investigate the effects of intravesical sequential treatments with BCG and MMC/ADM on immune-related cells, IHC staining for three markers (CD56, CD204 and Foxp3) was performed. Representative images of antibody-stained resected bladders are presented in Fig. 2 and the results are summarized in Table I. NK cells were significantly induced by treatment regimens containing $\mathrm{BCG}$ and were reduced by treatment with MMC monotherapy, compared with the control (BCG, P=0.0049; MMC-BCG, P=0.0048; ADM-BCG, $\mathrm{P}=0.0062$; MMC, $\mathrm{P}=0.039$; and $\mathrm{ADM}, \mathrm{P}=0.16$; Fig. $3 \mathrm{~A})$. NK cell levels were highest after treatment with BCG monotherapy, compared to all other treatment groups. Although NK cells were not recruited after treatment with MMC/ADM monotherapy, NK cells were significantly induced in the tumor microenvironment by sequential treatment with BCG 


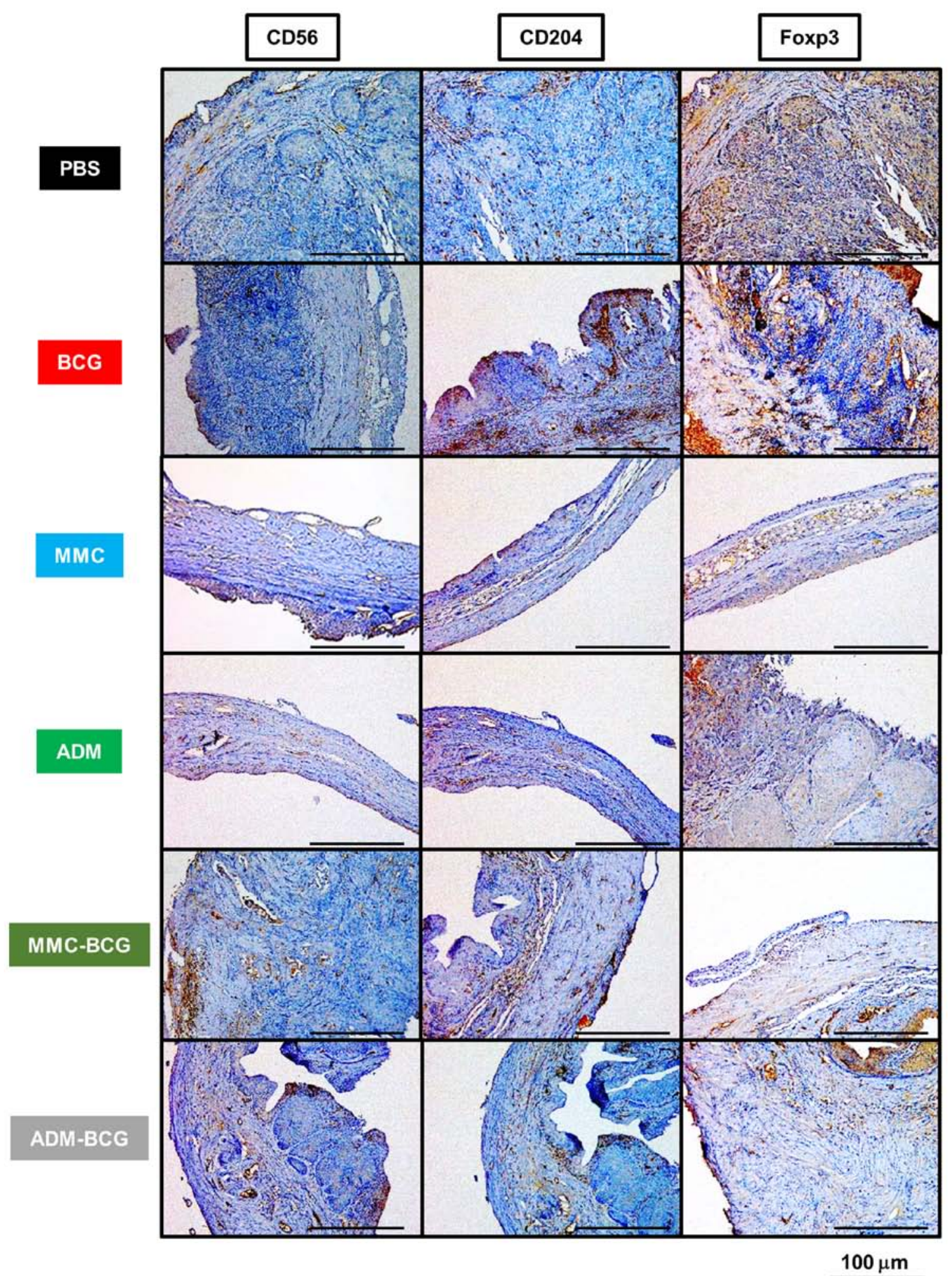

Figure 2. Representative images of bladder samples from each treatment group stained with three immunological markers. The expression levels of CD56 were increased in mice treated with BCG-containing regimens, and the expression levels of CD204 and Foxp3 were decreased in mice treated with chemotherapeutic agent-containing regimens. BCG, bacillus Calmette-Guerin.

and MMC/ADM (MMC-BCG, $\mathrm{P}=0.0048$; and ADM-BCG, $\mathrm{P}=0.015$; Fig. 3A). TAMs were significantly reduced by the treatment regimens containing chemotherapeutic agents, compared with the control (MMC, $\mathrm{P}=0.0043$; $\mathrm{ADM}, \mathrm{P}=0.0022$; MMC-BCG, $\mathrm{P}=0.010$; and ADM-BCG, $\mathrm{P}=0.0021$; Fig. 3B). Although TAMs were not reduced by the treatment with
BCG monotherapy, the sequential treatment with BCG and MMC/ADM reduced TAMs, indicating that intravesical treatment with chemotherapeutic agents prevented the recruitment of TAMs and/or killed TAMs in the tumor microenvironment. With regard to Tregs, treatment regimens containing ADM, as well as treatment with MMC monotherapy, significantly 
A

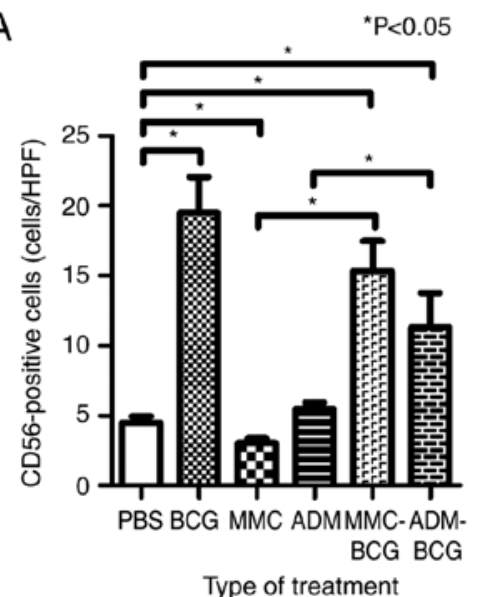

$B$

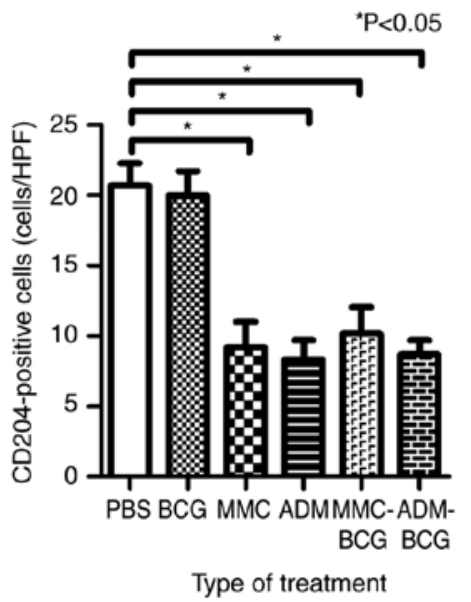

C

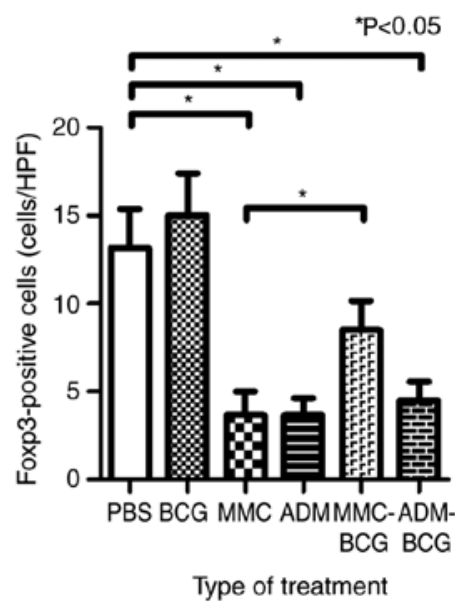

Figure 3. Relative expression levels of each immunological marker in treatment groups and PBS control. (A) CD56 was used as an NK cell marker. NK cells were induced by BCG-containing regimens and reduced by MMC alone. (B) CD204 was used for marking TAMs. TAMs were reduced by chemotherapeutic agent-containing regimens. (C) Foxp3 was used as a Treg marker. Tregs were reduced by MMC alone and ADM-containing regimens. "P<0.05. PBS, phosphate-buffered saline; NK, natural killer; BCG, bacillus Calmette-Guerin; MMC, mitomycin C; TAMs, tumor-associated macrophages; Treg, regulatory T cell; ADM, adriamycin.

A

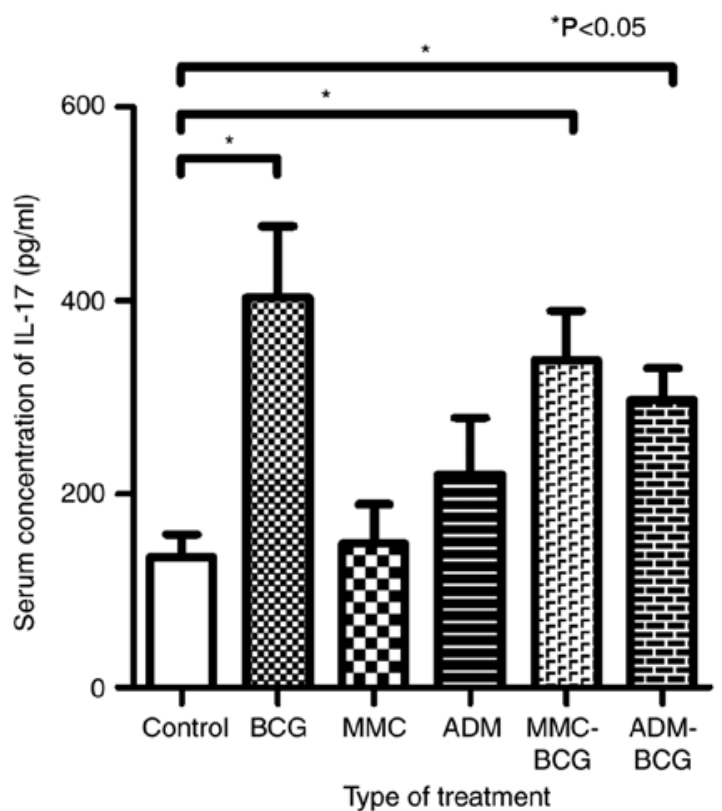

B

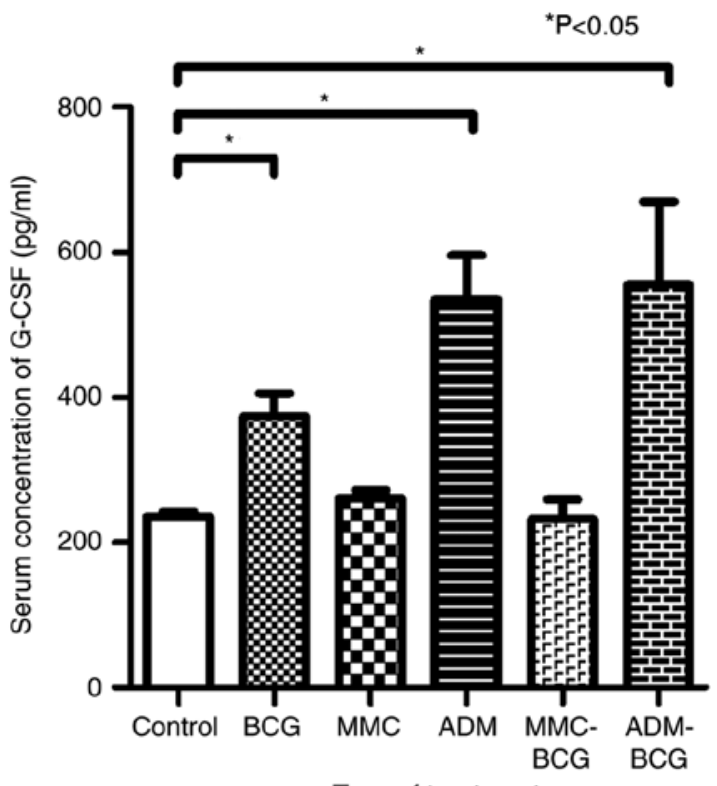

Type of treatment

Figure 4. Serum IL-17 and G-CSF concentrations by ELISA analysis. (A) IL-17 was increased after treatment with BCG-containing regimens compared with PBS control. (B) G-CSF was increased after treatment with BCG alone and ADM-containing regimens compared with PBS control. "P<0.05. IL, interleukin; G-CSF, granulocyte-colony stimulating factor; ELISA, enzyme-linked immunosorbent assay; BCG, bacillus Calmette-Guerin; ADM, adriamycin; PBS, phosphate-buffered saline.

reduced Tregs in the tumor microenvironment, compared with the control (ADM, P=0.0064; ADM-BCG, $\mathrm{P}=0.010$; and MMC, $\mathrm{P}=0.016$; Fig. 3C). Similar to TAMs, Tregs tended to be reduced after treatment with chemotherapeutic agents.

Systemic changes of $I L-17$ and G-CSF levels in serum caused by intravesical sequential treatment with $B C G$ and chemotherapeutic agents. To investigate the association between intravesical treatment and systemic changes to IL-17 and G-CSF levels, ELISA was performed using the serum obtained from the euthanized mice. The serum concentration of IL-17 was increased by treatment with BCG, MMC-BCG, and
ADM-BCG, compared with the control $(\mathrm{P}=0.0043, \mathrm{P}=0.0042$ and $\mathrm{P}=0.0087$, respectively; Fig. $4 \mathrm{~A})$. The treatment regimens containing BCG had an effect on the systemic induction of IL-17, indicating that instillation of BCG is an important factor for induction of IL-17, systemically. In contrast, the serum concentration of G-CSF was increased by treatment with BCG, ADM, and ADM-BCG, compared with the control ( $\mathrm{P}=0.0087, \mathrm{P}=0.0022$, and $\mathrm{P}=0.0021$, respectively; Fig. 4B). The treatment regimens containing ADM exhibited greater increase of G-CSF, indicating that instillation of ADM was a key factor for induction of G-CSF, systemically. The results are summarized in Table I. 


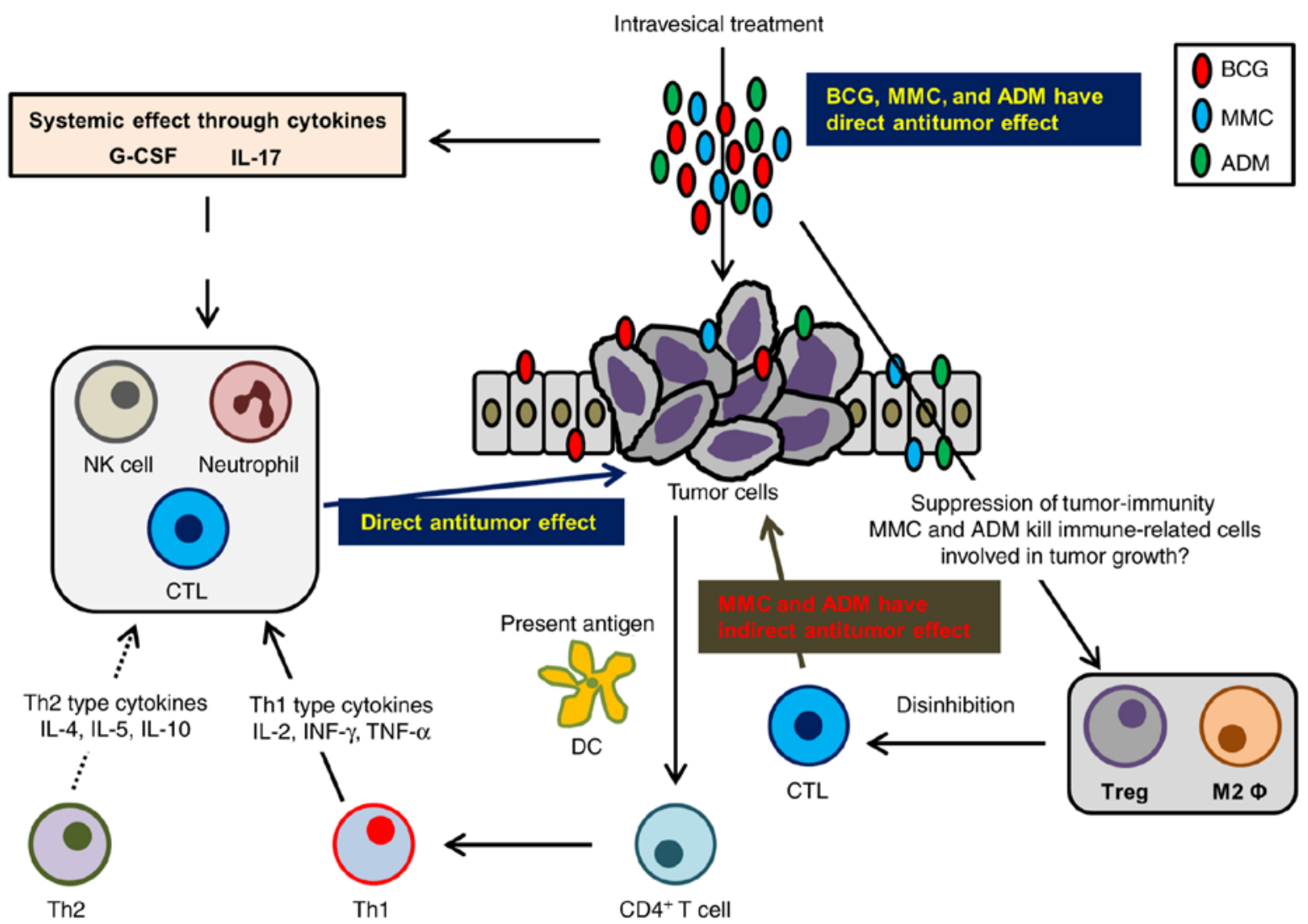

Figure 5. Schematic summary of the study. Intravesical treatment with chemotherapeutic agents demonstrates antitumor activity through direct cytotoxicity and indirectly through modulation of antitumor immunity. CTLs recognize cancer-specific antigens from apoptotic cancer cells and eliminate them. In addition, $\mathrm{DC}$ capture these antigens and present them to $\mathrm{CD} 4^{+} \mathrm{T}$ cells. Activated $\mathrm{CD} 4^{+} \mathrm{T}$ cells produce cytokines resulting in the activation of Th- 1 cells and the recruitment of CTLs, NK cells, and macrophages. The treatment with BCG enhances this pathway through activation of CTLs and NK cells. In contrast, intravesical treatment with chemotherapeutic agents modifies the tumor microenvironment to consist of less immunosuppressive cells, including a reduction in TAMs and Tregs. As a result, intravesical treatment with BCG works more effectively. IL-17 and G-CSF are induced by intravesical treatment, recruiting neutrophils to the tumor microenvironment. Neutrophils eliminate tumor cells by activating apoptotic pathways. Intravesical treatment with BCG and chemotherapeutic agents can be a novel treatment strategy for patients with BCG-failure non-muscle invasive bladder cancer by immunomodulation of the tumor microenvironment. CTLs, cytotoxic T cells; DC, dendritic cells; Th-1, helper T1; NK, natural killer; TAMs, tumor-associated macrophages; Tregs, regulatory T cells; BCG, bacillus Calmette-Guerin; IL, interleukin; G-CSF, granulocyte-colony stimulating factor.

\section{Discussion}

The present study revealed that intravesical sequential treatment with BCG and chemotherapeutic agents (MMC and ADM) elicited characteristic changes to immune cells in the tumor microenvironment of UCB. Although NK cells are induced by treatment with BCG monotherapy, treatment with chemotherapeutic agents followed by BCG also induced the recruitment of NK cells in the tumor microenvironment. The recruitment of TAMs and Tregs was inhibited by treatment with chemotherapeutic agents, except for treatment with MMC-BCG. Our previous study indicated that intravesical chemotherapy induced systemic changes in IL-17 and G-CSF levels, and that these cytokines had a role in promoting neutrophils, NK cells, and cytotoxic T lymphocytes (CTLs) in the tumor microenvironment, resulting in antitumor effects (23). Sequential treatment with BCG and chemotherapeutic agents, particularly ADM, induced IL-17 and G-CSF systemically. The summary of this study is displayed in Fig. 5. Intravesical sequential treatment with BCG and chemotherapeutic agents have direct antitumor effects upon T lymphocyte-dependent cytokines [particularly $\mathrm{T}$ helper 1 (Th-1) dependent cytokines such as IL-2,
IL-12, and interferon (INF)- $\gamma$ ], immune-related cells (NK cells, CTL and macrophages), and apoptotic pathways (tumor necrosis factor (TNF)-related apoptosis-inducing ligand (TRAIL) and fatty-acid synthase (Fas) ligand) (26). BCG treatment may also have indirect antitumor effects induced by chemotherapeutic agents, including the modulation of the tumor environment via the reduction of TAMs and Tregs.

Table II shows a summary of previous prospective studies involving intravesical sequential treatment with BCG and chemotherapeutic agents for patients with NMIBC (10-17,19-21,27-32). Intravesical sequential treatment has been studied since the 1990s, with the studies mainly focusing on the effects of chemotherapeutic agents, such as direct antitumor effects, tissue-scarifying of the bladder surface for attachment of BCG to the bladder wall, and the reduction of adverse events induced by BCG. Solsona et al reported that the 5-year disease-free interval (DFI) of sequential treatment with BCG and MMC, and treatment with BCG monotherapy, was 20.6 and $3.9 \%$, respectively. This was the largest study of its kind and revealed that treatment with BCG monotherapy had a long disease-free interval and a low rate of adverse events, compared with sequential treatment with BCG and MMC (11). In contrast, Di Stasi et al, which had the longest follow-up 


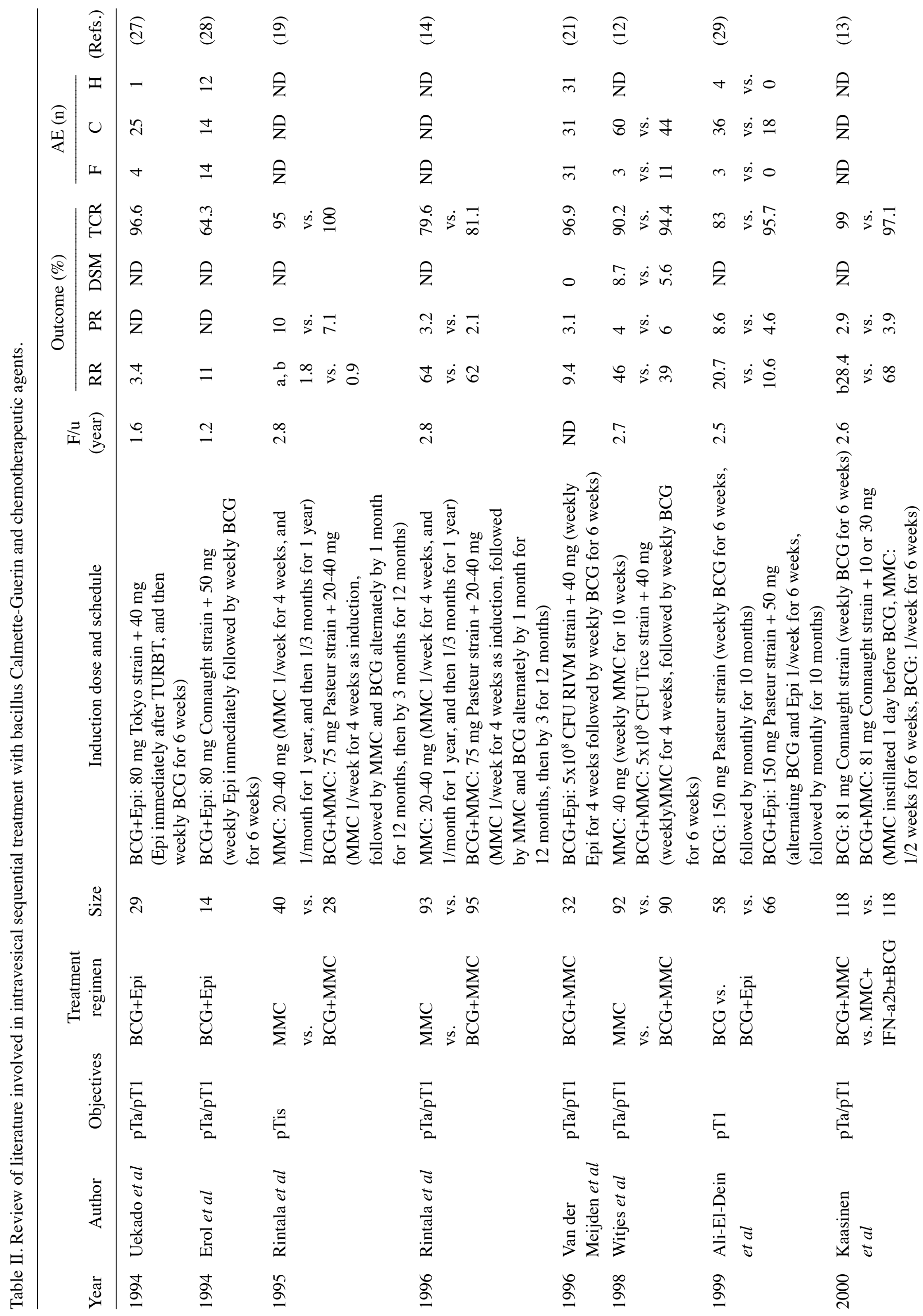




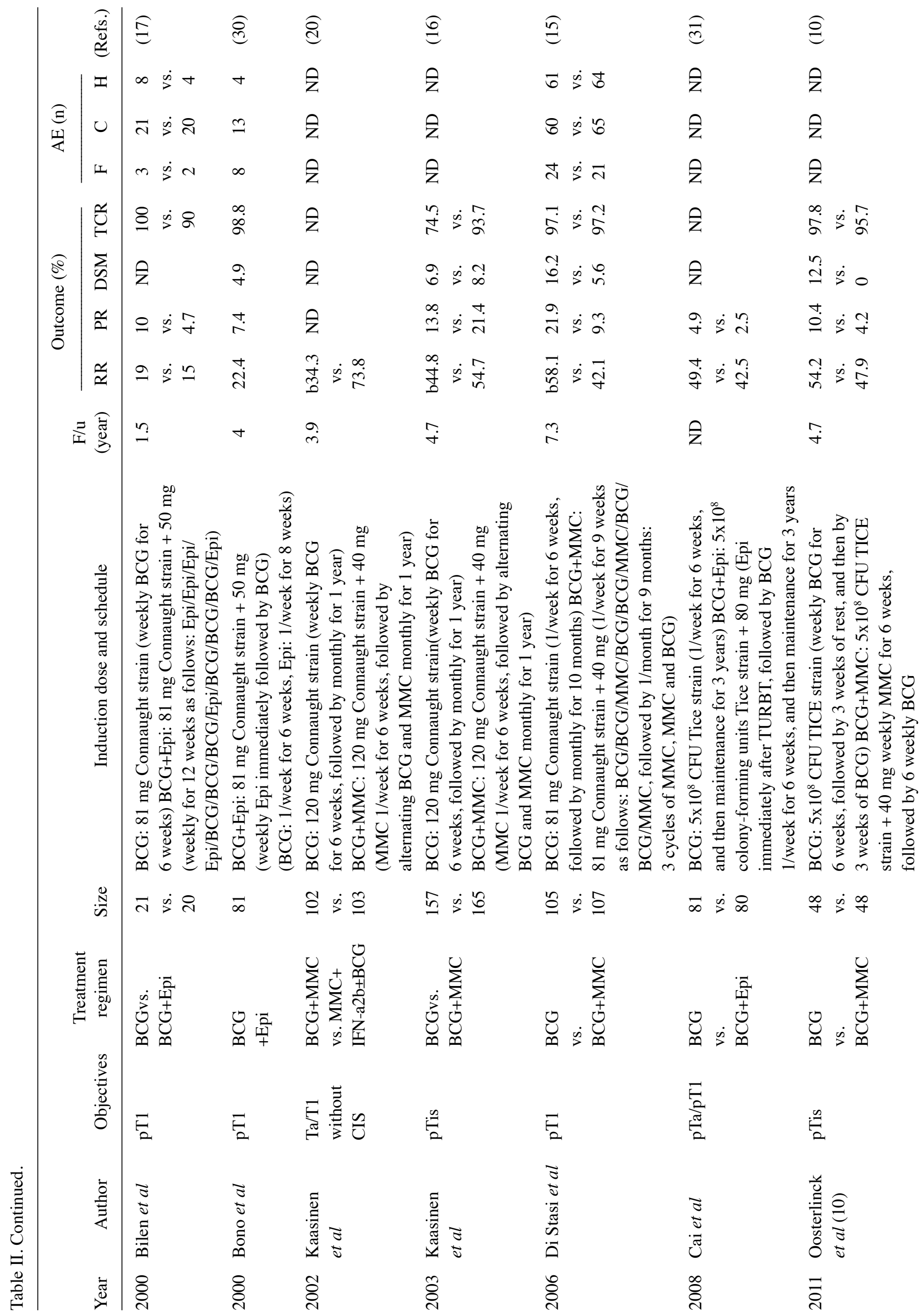




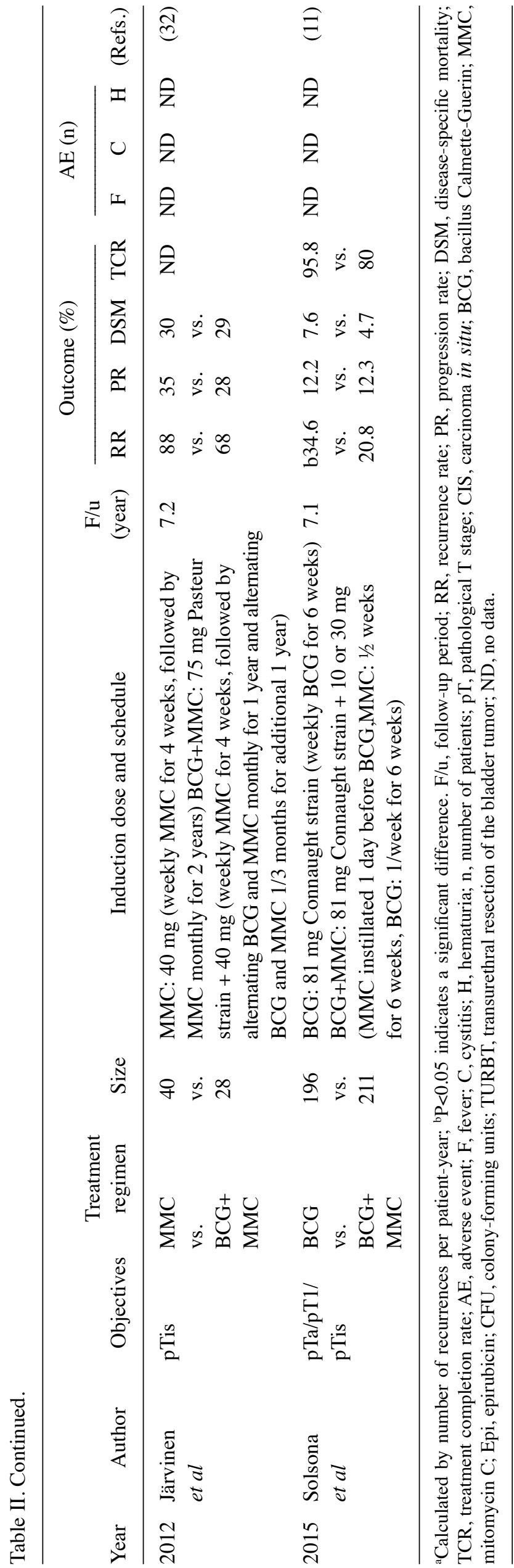

period, reported that DFI was significantly increased in patients treated sequentially with $\mathrm{BCG}$ and $\mathrm{MMC}$, compared to treatment with BCG monotherapy (15). The extent of the antitumor activity and decrease in adverse events following intravesical sequential treatment remains unclear due to the controversial results of each study. The antitumor effects of chemotherapeutic agents are mainly demonstrated by blocking DNA synthesis, RNA synthesis, or cell division. Our hypothesis was that intravesical treatment with chemotherapeutic agents modified the tumor microenvironment to conditions in which tumor cells found it difficult to proliferate and/or host immune-related cell attacks on tumor cells. Previous studies focusing on aspects involving immunomodulation of the tumor microenvironment are limited. The reduction of TAMs and Tregs, along with the potential effects of chemotherapeutic agents such as direct cytotoxicity and tissue-scarifying, demonstrated antitumor effects. Ghiringhelli et al reported that pretreatment with cyclophosphamide, which suppresses Tregs, enhanced the effects of subsequent immunotherapy in colorectal cancer (33). The treatment schedules of previous reports varied widely. It is our opinion, that the administration of MMC or ADM followed by BCG would be a better schedule, and that treatment should be repeated for several cycles. In addition, most of the previous prospective randomized trials were focused on sequential treatment with BCG and MMC, thus sequential treatment with BCG and ADM has not yet been verified by randomized trials. Our results indicated that sequential treatment with BCG and ADM inhibited the recruitment of Tregs to the tumor microenvironment, compared with BCG and MMC treatment. Verification of combination treatment with BCG and ADM is therefore necessary in the near future.

In the tumor microenvironment, activated Th- 1 produces immune-activating cytokines such as IL-2 and INF- $\gamma$, while stimulated CTLs play an important role in tumor immunity. Contrary to this, tumor cells produce immunosuppressive cytokines such as IL-10 and TNF- $\beta$ and induce immunosuppressive cells such as TAMs, Tregs, and myeloid-derived suppressor cells (MDSCs) (26,34-36). Various previous studies have suggested that intravesical chemotherapy modifies the tumor microenvironment into a less immunosuppressive condition, involving the induction of TAMs towards antitumoral M1 macrophages, proliferation of CTLs, an increase in the permeability of tumor cells to CTL-produced granzyme $\mathrm{B}$, selective reduction of MDSCs, and recruitment of NK cells and CTLs expressing perforin, granzyme B, and IFN- $\gamma$ (37-40). Treatment for patients with BCG failure is still challenging. High numbers of TAMs and Tregs in bladder tissue have been suggested to have a substantial correlation with BCG-failure in patients with $\operatorname{NMIBC}(22,23)$. Intravesical treatment with MMC and ADM prior to BCG can be a novel strategy for patients with high numbers of Tregs and TAMs in their transurethrally resected tissue.

With regard to the cytokines evaluated in the present study, the role of IL-17 in cancer remains unclear. The balance of Th-1, Th-2, and Th-17, which produce IL-17, is important for antitumor activity. Treatment with BCG was revealed to induce IL-17 and Th-17, resulting in antitumor activity (41). G-CSF could also be a marker for intravesical recurrence after intravesical treatment with BCG (42). Intravesical sequential treatment with BCG and chemotherapeutic agents induced IL-17 and G-CSF in this study. Thus, the increase in these cytokines leads to the 
subsequent induction of neutrophils and facilitates antitumor activity through Fas and/or TRAIL apoptotic pathways. In addition, these cytokines could be involved in immunomodulation via a reduction in immunosuppressive cells.

The present study has some limitations. The administration of BBN was not by oral gavage but was received in drinking water. Thus, the BBN intake varied among the mice and this could lead to variability in the tumor size and/or tumor behavior of each mouse. A confirmatory study with human bladder cancer specimens should be performed. A treatment schedule, controlling the timing of administration of BCG and chemotherapeutic agents, the number of treatment cycles, and the necessity of maintenance treatment, should be considered to achieve maximum efficacy of intravesical combination treatment. Further experiments are required to establish a novel treatment strategy for patients with NMIBC, particularly for patients with BCG-failure, based on immunological and molecular aspects.

In conclusion, intravesical sequential treatment with BCG and chemotherapeutic agents has more effective antitumor activity than that of BCG or chemotherapeutic agent monotherapy in a BBN-induced bladder cancer mouse model. Intravesical chemotherapy modified the tumor microenvironment to suppress pro-tumoral immunity and enhance antitumor immunity, increasing the efficiency of BCG treatment. These findings indicated that intravesical combination treatment could suppress resistance to BCG through enhancement of antitumor immunity (induction of NK cells) and inhibition of pro-tumoral immunity (reduction of TAMs and Tregs) and may be a novel strategy for BCG-failure NMIBC. Further studies, including clinical trials, may be required to establish appropriate strategies for intravesical sequential treatment, based on the immunomodulation of the tumor microenvironment.

\section{Acknowledgements}

We thank Mrs. Aya Asano (Department of Pathology, Nara Medical University, Nara, Japan) for giving us substantial help with the intravesical treatment.

\section{Funding}

The present study was supported by the JSPS KAKENHI grant nos. 15K10605 (KF) and 16K20159 (MM), and the Fiscal Years 2015-2016 Nara Medical University Grant-in-Aid for Collaborative Research Projects (KF and MM).

\section{Availability of data and materials}

The datasets generated and/or analyzed during the present study are not publicly available due to our hospital policy, but are available from the corresponding author upon reasonable request.

\section{Authors' contributions}

$\mathrm{SH}, \mathrm{MM}$ and $\mathrm{KF}$ contributed to the design of the study and writing of the manuscript. SH, YT, YM, SO, KO, KI, DG, and YI carried out the animal experiments and molecular biology studies. MM, YN, and NT performed the statistical tests. MM and $\mathrm{KF}$ assisted with the writing of the manuscript. MM and
YT reviewed the pathological diagnosis of bladder tissues. All authors read and approved the manuscript and agree to be accountable for all aspects of the research in ensuring that the accuracy or integrity of any part of the work are appropriately investigated and resolved.

\section{Ethics approval and consent to participate}

The Ethics Committee on Animal Research of Nara Medical University (Kashihara, Japan) approved this animal study. The reference number is 11649 .

\section{Patient consent for publication}

Not applicable.

\section{Competing interests}

The authors declare that they have no competing interests.

\section{References}

1. Siegel RL, Miller KD and Jemal A: Cancer statistics, 2016. CA Cancer J Clin 66: 7-30, 2016.

2. Editorial board of the cancer statistics in japan. Cancer statistic in Japan 2017. Tokyo: Foundation for promotion of cancer research; 2017. https://ganjoho.jp/data/reg_stat/statistics/brochure/2017/cancer_statistics_2017.pdf. Accessed 16 July, 2018.

3. Babjuk M, Burger M, Zigeuner R, Shariat SF, van Rhijn BW, Compérat E, Sylvester RJ, Kaasinen E, Böhle A, Palou Redorta J, et al: EAU guidelines on non-muscle-invasive urothelial carcinoma of the bladder: Update 2013. Eur Urol 64: 639-653, 2013.

4. Miyake M, Fujimoto K and Hirao Y: Active surveillance for nonmuscle invasive bladder cancer. Investig Clin Urol 57 (Suppl 1): S4-S13, 2016.

5. Herr HW: Intravesical bacillus Calmette-Guerin outcomes in patients with bladder cancer and asymptomatic bacteriuria. J Urol 187: 435-437, 2012.

6. Shahin O, Thalmann GN, Rentsch C, Mazzucchelli L and Studer UE: A retrospective analysis of 153 patients treated with or without intravesical bacillus Calmette-Guerin for primary stage T1 grade 3 bladder cancer: Recurrence, progression and survival. J Urol 169: 96-100, 2003.

7. Herr HW and Morales A: History of Bacillus Calmette-Guerin and bladder cancer: An immunotherapy success story. J Urol 179: 53-56, 2008.

8. Lamm DL, Blumenstein BA, Crissman JD, Montie JE, Gottesman JE, Lowe BA, Sarosdy MF, Bohl RD, Grossman HB, Beck TM, et al: Maintenance bacillus Calmette-Guérin immunotherapy for recurrent TA, T1 and carcinoma in situ transitional cell carcinoma of the bladder: A randomized Southwest Oncology Group Study. J Urol 163: 1124-1129, 2000.

9. Nakai Y, Anai S, Tanaka N, Chihara Y, Haramoto M, Otani T, Nakagawa Y, Hirao Y, Konishi N and Fujimoto K: Insignificant role of bacillus Calmette-Guérin maintenance therapy after complete transurethral resection of bladder tumor for intermediate- and high-risk non-muscle-invasive bladder cancer: Results from a randomized trial. Int J Urol 23: 854-860, 2016.

10. Oosterlinck W, Kirkali Z, Sylvester R, da Silva FC, Busch C, Algaba F, Collette S and Bono A: Sequential intravesical chemoimmunotherapy with mitomycin $\mathrm{C}$ and bacillus Calmette-Guérin and with bacillus Calmette-Guérin alone in patients with carcinoma in situ of the urinary bladder: Results of an EORTC genito-urinary group randomized phase 2 trial (30993). Eur Urol 59: 438-446, 2011.

11. Solsona E, Madero R, Chantada V, Fernandez JM, Zabala JA, Portillo JA, Alonso JM, Astobieta A, Unda M, Martinez-Piñeiro L, et al: Sequential combination of mitomycin $\mathrm{C}$ plus bacillus Calmette-Guérin (BCG) is more effective but more toxic than BCG alone in patients with non-muscle-invasive bladder cancer in intermediate- and high-risk patients: Final outcome of CUETO 93009, a randomized prospective trial. Eur Urol 67: 508-516, 2015. 
12. Witjes JA, Caris CT, Mungan NA, Debruyne FM and Witjes WP Results of a randomized phase III trial of sequential intravesical therapy with mitomycin $\mathrm{C}$ and bacillus Calmette-Guerin versus mitomycin $\mathrm{C}$ alone in patients with superficial bladder cancer. J Urol 160: 1668-1671, 1998.

13. Kaasinen E, Rintala E, Pere AK, Kallio J, Puolakka VM, Liukkonen T and Tuhkanen K: Weekly mitomycin C followed by monthly bacillus Calmette-Guerin or alternating monthly interferon-alpha2B and bacillus Calmette-Guerin for prophylaxis of recurrent papillary superficial bladder carcinoma. J Urol 164: 47-52, 2000

14. Rintala E, Jauhiainen K, Kaasinen E, Nurmi M and Alfthan O: Alternating mitomycin $\mathrm{C}$ and bacillus Calmette-Guerin instillation prophylaxis for recurrent papillary (stages Ta to T1) superficial bladder cancer. Finnbladder Group. J Urol 156: 56-60, 1996.

15. Di Stasi SM, Giannantoni A, Giurioli A, Valenti M, Zampa G, Storti L, Attisani F, De Carolis A, Capelli G, Vespasiani G, et al: Sequential BCG and electromotive mitomycin versus BCG alone for high-risk superficial bladder cancer: A randomised controlled trial. Lancet Oncol 7: 43-51, 2006.

16. Kaasinen E, Wijkström H, Malmström PU, Hellsten S, Duchek M, Mestad O and Rintala E; Nordic Urothelial Cancer Group: Alternating mitomycin $\mathrm{C}$ and $\mathrm{BCG}$ instillations versus $\mathrm{BCG}$ alone in treatment of carcinoma in situ of the urinary bladder: A nordic study. Eur Urol 43: 637-645, 2003.

17. Bilen CY, Ozen H, Aki FT, Aygün C, Ekici S and Kendi S Clinical experience with BCG alone versus BCG plus epirubicin. Int J Urol 7: 206-209, 2000.

18. Hilton WM, Ercole B, Parekh DJ, Sonpavde G, Ghosh R and Svatek RS: Efficacy of combined intravesical immunotherapy and chemotherapy for non-muscle invasive bladder cancer. Expert Rev Anticancer Ther 11: 949-957, 2011.

19. Rintala E, Jauhiainen K, Rajala P, Ruutu M, Kaasinen E and Alfthan O: Alternating mitomycin $\mathrm{C}$ and bacillus Calmette-Guerin instillation therapy for carcinoma in situ of the bladder. The Finnbladder Group. J Urol 154: 2050-2053, 1995.

20. Kaasinen E, Rintala E, Hellström P, Viitanen J, Juusela H, Rajala P, Korhonen H and Liukkonen T; FinnBladder Group: Factors explaining recurrence in patients undergoing chemoimmunotherapy regimens for frequently recurring superficial bladder carcinoma 1. Eur Urol 42: 167-174, 2002

21. Van der Meijden AP, Hall RR, Macaluso MP, Pawinsky A, Sylvester R and Van Glabbeke M: Marker tumour responses to the sequential combination of intravesical therapy with mitomycin-C and BCG-RIVM in multiple superficial bladder tumours. Report from the European Organisation for Research and Treatment on Cancer-Genitourinary Group (EORTC 30897). Eur Urol 29: 199-203, 1996

22. Miyake M, Tatsumi Y, Gotoh D, Ohnishi S, Owari T, Iida K, Ohnishi K, Hori S, Morizawa Y, Itami Y, et al: Regulatory $\mathrm{T}$ cells and tumor-associated macrophages in the tumor microenvironment in non-muscle invasive bladder cancer treated with intravesical bacille Calmette-Guérin: A long-term follow-up study of a Japanese cohort. Int J Mol Sci 18: E2186, 2017.

23. Hori S, Miyake M, Tatsumi Y, Onishi S, Morizawa Y, Nakai Y, Tanaka $\mathrm{N}$ and Fujimoto K: Topical and systemic immunoreaction triggered by intravesical chemotherapy in an N-butyl-N-(4-hydroxybutyl) nitorosamine induced bladder cancer mouse model. PLoS One 12: e0175494, 2017

24. Hori S, Miyake M, Tatsumi Y, Morizawa Y, Nakai Y, Onishi S, Onishi $\mathrm{K}$, Iida $\mathrm{K}$, Gotoh D, Tanaka $\mathrm{N}$ and Fujimoto $\mathrm{K}$ : Gamma-Klotho exhibits multiple roles in tumor growth of human bladder cancer. Oncotarget 9: 19508-19524, 2018.

25. Hori S, Miyake M, Onishi S, Tatsumi Y, Morizawa Y, Nakai Y, Anai S, Tanaka $\mathrm{N}$ and Fujimoto $\mathrm{K}$ : Clinical significance of $\alpha$ - and $\beta$-Klotho in urothelial carcinoma of the bladder. Oncol Rep 36: 2117-2125, 2016.

26. Kitamura $\mathrm{H}$ and Tsukamoto $\mathrm{T}$ : Immunotherapy for urothelial carcinoma: Current status and perspectives. Cancers 3: 3055-3072, 2011.

27. Uekado Y, Hirano A, Shinka T and Ohkawa T: The effects of intravesical chemoimmunotherapy with epirubicin and bacillus Calmette-Guérin for prophylaxis of recurrence of superficial bladder cancer: A preliminary report. Cancer Chemother Pharmacol 35 (Suppl): S65-S68, 1994.
28. Erol A, Ozgür S, Basar M and Cetin S: Trial with bacillus Calmette-Guérin and epirubicin combination in the prophylaxis of superficial bladder cancer. Urol Int 52: 69-72, 1994.

29. Ali-El-Dein B, Nabeeh A, Ismail EH and Ghoneim MA: Sequential bacillus Calmette-Guerin and epirubicin versus bacillus Calmette-Guerin alone for superficial bladder tumors: A randomized prospective study. J Urol 162: 339-342, 1999.

30. Bono AV, Lovisolo JA and Saredi G: Transurethral resection and sequential chemo-immunoprophylaxis in primary T1G3 bladder cancer. Eur Urol 37: 478-483, 2000.

31. Cai T, Nesi G, Tinacci G, Zini E, Mondaini N, Boddi V, Mazzoli S and Bartoletti R: Can early single dose instillation of epirubicin improve bacillus Calmette-Guerin efficacy in patients with nonmuscle invasive high risk bladder cancer? Results from a prospective, randomized, double-blind controlled study. J Urol 180: 110-115, 2008

32. Järvinen R, Kaasinen E, Rintala E and Group TF: Long-term results of maintenance treatment of mitomycin $\mathrm{C}$ or alternating mitomycin $\mathrm{C}$ and bacillus Calmette-Guérin instillation therapy of patients with carcinoma in situ of the bladder: A subgroup analysis of the prospective FinnBladder 2 study with a 17-year follow-up. Scand J Urol Nephrol 46: 411-417, 2012.

33. Ghiringhelli F, Larmonier N, Schmitt E, Parcellier A, Cathelin D, Garrido C, Chauffert B, Solary E, Bonnotte B and Martin F: $\mathrm{CD} 4{ }^{+} \mathrm{CD} 25^{+}$regulatory $\mathrm{T}$ cells suppress tumor immunity but are sensitive to cyclophosphamide which allows immunotherapy of established tumors to be curative. Eur J Immunol 34: 336-344, 2004.

34. Sakaguchi S, Sakaguchi N, Shimizu J, Yamazaki S, Sakihama T, Itoh M, Kuniyasu Y, Nomura T, Toda $M$ and Takahashi T: Immunologic tolerance maintained by $\mathrm{CD} 25^{+} \mathrm{CD} 4^{+}$regulatory T cells: Their common role in controlling autoimmunity, tumor immunity, and transplantation tolerance. Immunol Rev 182: 18-32, 2001.

35. Gabrilovich DI and Nagarai S: Myeloid-derived suppressor cells as regulators of the immune system. Nat Rev Immunol 9: 162-174, 2009.

36. Zhao Y, Wang D, Xu T, Liu P, Cao Y, Wang Y, Yang X, Xu X, Wang $X$ and Niu H: Bladder cancer cells re-educate TAMs through lactate shuttling in the microfluidic cancer microenvironment. Oncotarget 6: 39196-39210, 2015.

37. Svatek RS, Zhao XR, Morales EE, Jha MK, Tseng TY, Hugen CM, Hurez V, Hernandez J and Curiel TJ: Sequential intravesical mitomycin plus Bacillus Calmette-Guérin for non-muscle-invasive urothelial bladder carcinoma: Translational and phase I clinical trial. Clin Cancer Res 21: 303-311, 2015.

38. Obeid M, Tesniere A, Ghiringhelli F, Fimia GM, Apetoh L, Perfettini JL, Castedo M, Mignot G, Panaretakis T, Casares N, et al: Calreticulin exposure dictates the immunogenicity of cancer cell death. Nat Med 13: 54-61, 2007.

39. Ramakrishnan R, Assudani D, Nagaraj S, Hunter T, Cho HI, Antonia S, Altiok S, Celis E and Gabrilovich DI: Chemotherapy enhances tumor cell susceptibility to CTL-medeated killing during cancer immunotherapy in mice. J Clin Invest 120: 1111-1124, 2010

40. Alizadeh D, Trad M, Hanke NT, Larmonier CB, Janikashvili N, Bonnotte B, Katsanis E and Larmonier N: Doxorubicin eliminates myeloid-derived suppressor cells and enhances the efficacy of adoptive T-cell transfer in breast cancer. Cancer Res 74: 104-118, 2014.

41. Saban MR, Simpson C, Davis C, Wallis G,Knowlton N,Frank MB, Centola M, Gallucci RM and Saban R: Discriminators of mouse bladder response to intravesical Bacillus Calmette-Guerin (BCG). BMC Immunol 8: 6, 2007.

42. Shintani Y, Sawada Y, Inagaki T, Kohjimoto Y, Ueoka Y and Shinka T: Intravesical instillation therapy with bacillus Calmette-Guérin for superficial bladder cancer: Study of the mechanism of bacillus Calmette-Guérin immunotherapy. Int J Urol 14: 140-146, 2007. 